\title{
Locating Corticospinal Neurons by Retrograde Axonal Transport of Horseradish Peroxidase ${ }^{1}$
}

\author{
Samuel P. Hicks and Constance J. D'Amato \\ Department of Pathology, University of Michigan Medical Center, \\ Ann Arbor, Michigan 48109 \\ Received February 17, 1977
}

\begin{abstract}
Different views of the location and organization of corticospinal (CS) neurons in the rat have resulted from fiber degeneration studies, electrophysiologically derived somatotopic maps, studies of CS axonal branching, and functional alterations after ablation of parts of the motor-sensory cortex. To locate more precisely CS neurons that projected to different levels of the spinal cord, and to determine whether or not they were arranged somatotopically, we labeled them with horseradish peroxidase (HRP) from their cut spinal axons. These labeled neurons were arranged in a major caudal band about $4.5 \mathrm{~mm}$ long and $3 \mathrm{~mm}$ wide corresponding principally to areas 3,4 , and 6 , and a minor rostral band in the anterior part of area 10. The caudal band also corresponded to electrophysiologically derived somatotopic hindlimb and forelimb motor areas. The most significant finding was that CS neurons labeled from the lumbar spinal cord and from cervical levels were intermixed generally throughout the caudal band, showing virtually no somatotopic anatomic arrangement. The rostral band, which corresponded to mouth parts in somatotopic maps, was an unexpected finding. Calculations based on estimates of the numbers of CS axons at different cord levels indicated that about $10,000 \mathrm{CS}$ neurons in each cortex projected as far as the rostral cervical cord, and about 2000 continued as far as lumbar levels. The HRP method as used was capable of labeling a majority of the calculated numbers of CS neurons, but showed a large variance in the total numbers of ncurons labelcd. The distributions of the neurons within the domains of the bands was consistent regardless of the numbers labeled.
\end{abstract}

\section{INTRODUCTION}

Concepts of the corticospinal (CS) system have emphasized that individual neurons in the motor-sensory cortex influence particular muscles,

Abbreviations: CS-corticospinal, HRP-horseradish peroxidase, DAB-diaminobenzidine.

${ }^{1}$ This research is supported by U.S. Public Health Service Grant NS 10531. 
through spinal motoneuron pools, and in turn receive feedback from those muscles, and from the skin, joints, and tendons most closely associated with them. A virtual one-to-one central-peripheral relationship has been seen in those connections $(2,5,16)$. Neurophysiologically derived somatotopic maps of the motor-sensory (motor and somatosensory) cortex of the rat have been in accord with this view $(7,17,18)$. However, the anatomic projections from different parts of the rat's motor-sensory cortex, and the functional effects of ablating them, suggest that parts of this cortex also possess a nonsomatotopic organization for movements of multiple parts of the body and for different components of movement $(6,8)$. Moreover, recent neurophysiologic studies have shown that a substantial number of CS axons (as well as bulbospinal axons) give off branches and synapse at two or more levels in the brain stem and spinal cord, providing one-tomore than one central-peripheral relationships $(3,15)$. To find out whether the CS ncurons are arranged somatotopically or in some other configuration, and how the configuration develops postnatally, we began a study of these neurons in the rat using horseradish peroxidase (HRP) to label them from the cut ends of their axons at different spinal cord levels. This paper describes the location of CS neurons whose axons reach various levels of the spinal cord in the mature rat, gives some estimates of their numbers, and indicates approximately their positions in relation to published somatotopic, cytoarchitectural, and stereotaxic maps. Preliminary reports and demonstrations of some of the results have been made $(6,9,10)$.

\section{METHODS}

General Description. HRP was introduced into the site of a partial surgical transection of the rat's spinal cord that included the CS tracts at about the $\mathrm{Cl}, \mathrm{C}$, or $\mathrm{L} 1$ level. [The CS tract of the rat, one of the largest among mammals in proportion to brain size, runs in the base of the dorsal column of the spinal cord after decussating completely from the medullary pyramids (8).] After suitable intervals, the animals were killed and their nervous systems fixed by vascular perfusions for demonstration of HRP (14). Serial frozen microscopic sections of one sagittal half of the brain with the first segment of the spinal cord were always made; serial frontal sections of the forebrain of the other half were sometimes made. Peroxidase was usually demonstrated with diaminobenzidine (DAB) for dark-field microscopy; the benzidine blue reaction was also used with safranine or Darrow red counterstain. Some sections that included the site of cord transection were stained with hematoxylin and eosin. Distribution of the HRP-labeled cells was recorded and mapped, and the zones in which they were located were measured. Counts of the labeled cells were made in a number of cases. The total numbers of $\mathrm{CS}$ neurons whose axons reached various levels 
of the spinal cord were estimated by measuring the cross-sectional areas of the CS tracts at those levels, and assuming that the areas were proportional to the number of axons, extrapolating the value at each level from Bernstein's (4) figure of about 6000 axons at T8. The positions of the HRP-labeled neurons were superimposed on an outline of the rat's brain scaled in millimeters to show approximately the relations between their anatomic locations and certain electrophysiologically derived somatotopic areas $(7)$, cytoarchitectural cortical areas $(12,13)$, and stereotaxic coordinate maps (11).

Animals. We used male or female $\mathrm{F}_{1}$ Irish and hooded rats derived from crossing albino and black lines long brother-sister mated as previously described (8). Housing facilities and care of the animals were fully accredited by the American Association for Accreditation of Laboratory Animal Care, and conduct of the experiments conformed to standards in the Guide for the Care and Use of Laboratory Animals, U.S. Department of Health, Education, and Welfare publication 73-23, 1972.

Experimental Procedure. Operations were carried out under a microscope with the animal under ethcr ancsthesia, in an animal surgcry. The spinal cord was exposed and partially cut transversely with fine scissors. The extent of the transection was easily visualized because the wound immediately gaped. It included the posterior columns (with CS tracts), the center of the cord, parts of the posterior horns, and usually dorsomedial parts of the lateral columns. A pledget of HRP type II (Sigma) crystals usually weighing about 0.1 to $0.2 \mathrm{mg}$ was introduced into the cord incision where it dissolved instantly in the fluid in the wound. The skin incisions were closed with sutures. Rats with cervical lesions which took little food or water were given subcutaneous injections of physiologic saline with $5 \%$ glucose. Usually, the animals with cervical lesions were killed and fixation begun about $48 \mathrm{~h}$ after introduction of HRP, or about $72 \mathrm{~h}$ for those with lumbar lesions.

$\mathrm{HRP}$ was placed in incisions at $\mathrm{L} 1$ in six rats, at $\mathrm{C} 7$ in four rats, and at $\mathrm{C} 1$ in three rats, all 1 or 2 months old.

Histologic Methods. The serial sections were collected in order in seven, or in two cases five, samples. Counts of labeled neurons were usually made in one- or two-sevenths of the sections, but in one $\mathrm{L} 1$ and one $\mathrm{C} 1$ rat they were made in three-fifths of all the sections of one hemisphere. The number of labeled CS neurons in a hemisphere was computed from the sample and corrected by Abercrombie's (1) method. Sections were cut $40 \mu \mathrm{m}$ thick, treated to demonstrate HRP, dehydrated, cleared, and mounted. The mean thickness of sections and width of labeled neuron bodies after this procedure were, respectively, about $34 \mu \mathrm{m}(34.1 \pm 3.6 \mu \mathrm{m}, N=80)$ and $14 \mu \mathrm{m}(14.2$ $\pm 0.8 \mu \mathrm{m}, N=100$ ). Abercrombie's correction was 0.7 . The pole-to-pole 
reduction in length of the cerebral hemispheres in these preparations was from 15 to $16 \mathrm{~mm}$ in the fresh state to 10 to $11 \mathrm{~mm}$ after processing.

To estimate the total numbers of CS tract axons in various levels of the spinal cord, cross sections at about the level of L1, T4, C7, and C1 were prepared from rats 1 and 2 months old by a modified Mann's eosin-methyl blue stain for axis cylinders and myelin sheaths. The magnified sections were projected onto graph paper and the outlines of the CS tracts were traced to compare their areas at the different levels.

Locating the Labeled Corticospinal Neurons. To describe the locations of the neurons, the HRP microscopic sections were compared with the sections and diagrams in the stereotaxic maps of König and Klippel (11), the cytoarchitectural maps of Krieg $(12,13)$, and our own sets of serially sectioned brains of rats stained with luxol fast blue and cresyl violet, Cajal pyridine silver nitrate, and Golgi methods. The domains of the labeled neurons were also comparcd with those of the motor areas of Hall and Lindholm's (7) somatotopic maps. In those published studies, unfixed brains as well as brains fixed by various methods were used and correction factors applicd; thereforc, we used the outline of an unfixed mature rat brain in situ at autopsy in Fig. 3 and transferred the various data to it for the best fit possible.

\section{RESULTS}

Position of Labeled Corticospinal Neurons. Labeled neurons in the forebrain were situated exclusively in layer $\mathrm{V}$ of the dorsal isocortex (Figs. $1,2)$, and they formed two bands, separated by a gap: a major caudal hand in the parietal-frontal cortex $(12,13)$ and a minor rostral band farther frontally. The caudal band, the more densely populated, was approximately rectangular and resulted when labeling was done from $\mathrm{C} 1$, C7, or I.1. The rostral band, which was not sharply defined, appeared after labeling from $\mathrm{C1}$ or $\mathrm{C} 7$, but not L1. Labeled neurons were rare or absent in the gap between the bands. These bands are mapped in Fig. 3 as dotted rectangles on an outline of the rat's brain, on which are also superimposed some of Krieg's $(12,13)$ cytoarchitectural areas, Hall and Lindholm's (7) somatotopic motor areas, and millimeter scales from König and Klippel's (11) atlas of the rat's brain. Labeling occurred in neurons at all levels of layer $\mathrm{V}$ in both bands. The posterior border of the caudal band was in a frontal plane passing through about the middle of the dorsal hippocampus, corresponding to the plane between 3.5 and $4 \mathrm{~mm}$ rostral to 0 reference in König and Klippel; the plane of its anterior border was at 7.5 to $8 \mathrm{~mm}$ passing through the striatum. The medial border was about 0.5 $\mathrm{mm}$ lateral to the midline, in a sagittal plane passing just medial to the crest of the cingulum, and extended laterally 2.5 to a little more than $3 \mathrm{~mm}$ 

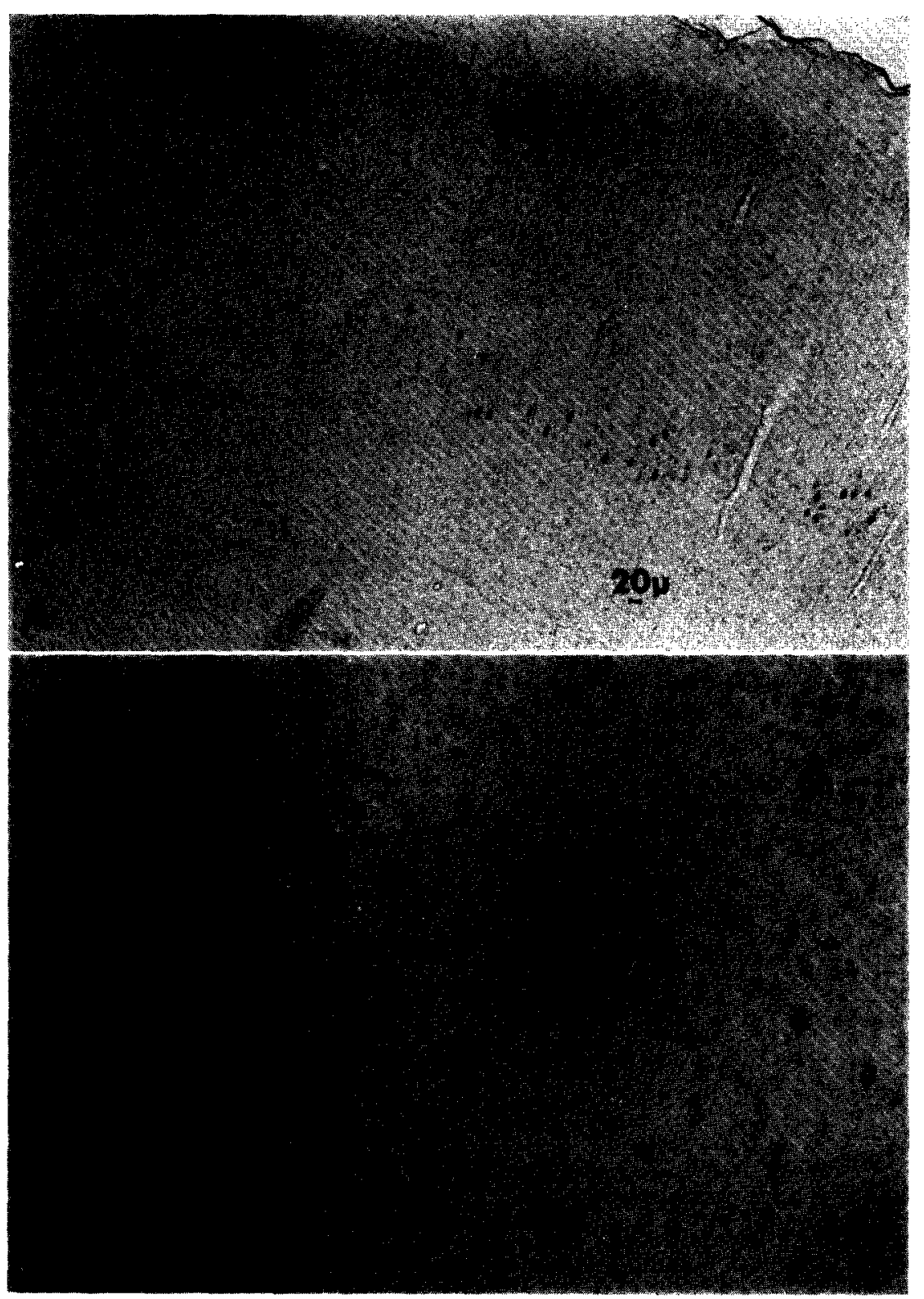

FIG. 1. A-Frontal section of the dorsomedial cortex of the rat's brain indicating the entire width of the caudal band of CS neurons. Heavily labeled neurons are black. The cingulum is at the left border. HRP was introduced at $\mathrm{Cl}$. (Benzidine blue and safranine.) B-Higher magnification of center of $\mathrm{A}$ to show heavily and lightly labeled neurons (arrows). There were 90 labeled neurons in this section of the band. 


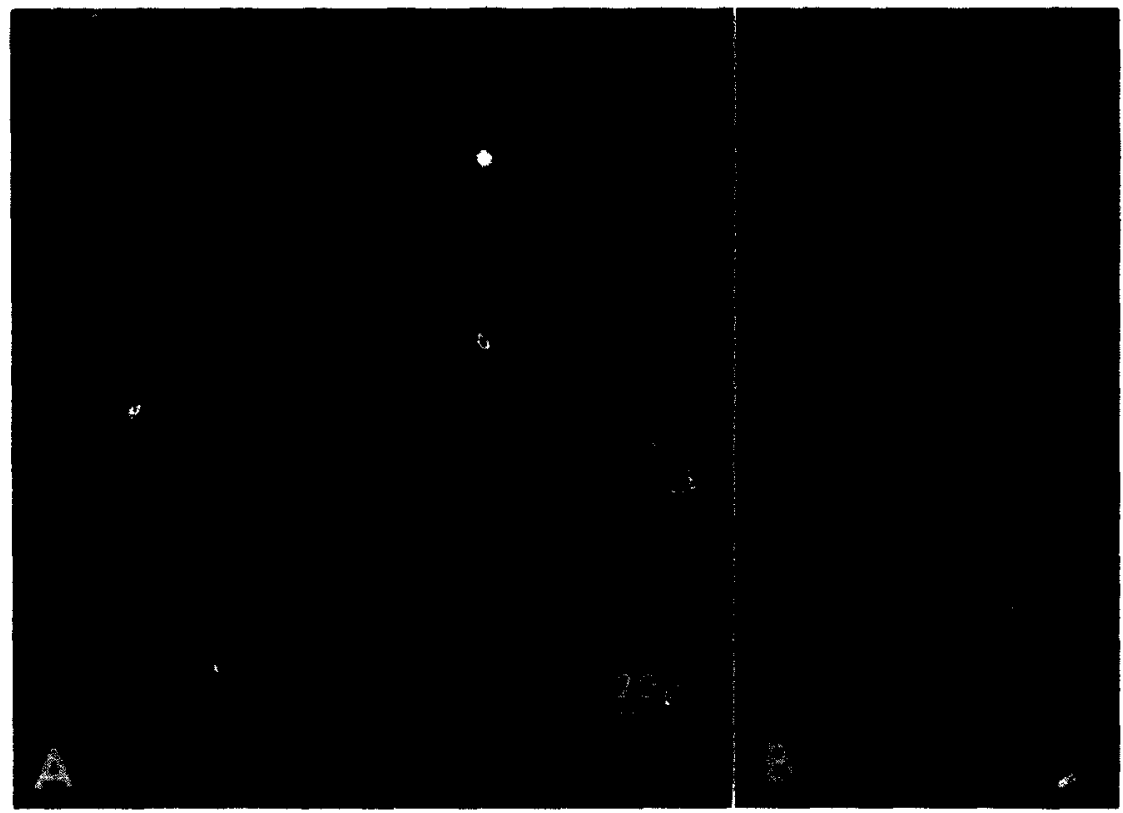

FIG. 2. A-CS neurons at the anterior border of the caudal band. Arrow indicates a neuron at level of sharpest focus. HRP was introduced at L1. White spots are residual red cells. (Dark field, DAB) B-CS neurons in the rostral band. Arrow indicates three labeled neurons at different levels of focus. HRP was introduced at C7. (Dark field, DAB)

from the midline. The outline of the caudal band in Fig. 3 represents the greatest extent of the area in which neurons labeled from either lumbar or cervical levels were found; the maximum length of the band in our finished HRP sections was about $3.3 \mathrm{~mm}$ or about $4.8 \mathrm{~mm}$ corrected for an unfixed brain. Matched with König and Klippel's coordinates it was a little shorter. The labeled neurons were distributed generally throughout the caudal band, with small exceptions described later, whether the HRP was introduced at the lumbar or cervical levels, but the total number varied with the level at which HRP was introduced.

Cytoarchitecturally, most of the labeled CS neurons appeared in Krieg's $(12,13)$ areas $3,4,6$, and the anterior part of 7 , as shown by matching the HRP sections against his descriptions and our serially sectioned normal brains. The apparent presence of labeled neurons in the adjacent borders of $29 \mathrm{c}, 18$, and 10 could be attributed to these regions being transition zones between areas, to limits of accuracy in delineating the caudal band, and to intrinsic errors in superimposing data from several sources on one map. The medial border of the caudal hand was sharp: The most medially situated neurons lay in the radiata of the cingulum in the junction of iso- 


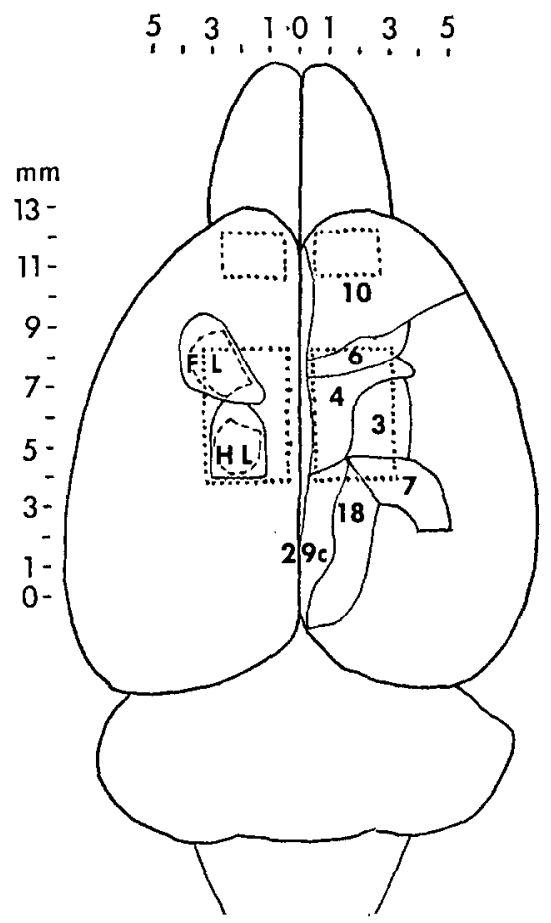

Fig. 3. The outline of an unfixed rat's brain measured in millimeters according to König and Klippel (11) where $7 \mathrm{~mm}$ on the longitudinal scale corresponds to a frontal plane passing through the bregma ( 0 reference on other maps). Relevant cytoarchitectural areas $(3,4,6$, etc.) of Krieg $(12,13)$ are drawn on the right and areas from which Hall and Lindholm (7) electrophysiologically elicited forelimb (FL) and hindlimb (HL) movements are shown on the left. The smaller dashed-line areas represent regions from which movements resulted from a $1-\mathrm{kHz}$ stimulus, and the larger solid-line areas, movements from $300 \mathrm{~Hz}$. Rectangles in dotted outline are rostral and caudal bands.

cortex and cingular cortex, their long axes directed upward and inward toward the midline.

The caudal band substantially overlapped the motor areas delineated by Hall and Lindholm (7), who prepared two maps as shown in Fig. 3. The smaller areas from which they elicited forelimb (FL) and hindlimb (HL) movements electrophysiologically with $1-\mathrm{kHz}$ stimuli are shown in dashed outline; the larger areas in solid line represent areas in which the movements followed stimuli of $300 \mathrm{~Hz}$.

Counts of Corticospinal Neurons. In two rats labeled from $\mathrm{C} 1$ there were 6500 and 3800 neurons in the caudal band and 500 and 270 in the rostral band, respectively. In two rats labeled from C7 there were 2440 and 3500 neurons in the caudal band and 50 and 400 in the rostral band. In four 
rats labeled from the L1 region, there were $2100,1440,840$, and 240 neurons in the caudal band. (The 2100 count from labeling from L1 and the 3800 count from labeling from $\mathrm{C} 1$ were based on three-fifths of all the sections from one half brain as noted under Methods.)

Experience with these and other current experiments showed that when counts (uncorrected) in the caudal band in a substantial number of sections ranged from about 90 to 150 , the total count was several thousand labeled neurons, a "high count;" about 40 to 90 indicated a total of 1000 to 2000 , an intermediate count;" and a few to 40 neurons indicated a total in the hundreds, a "low count." In the remaining animals in which counts of labeled neurons were not completed, it was possible by inspection to estimate whether the count would have been high, low, or intermediate. On this basis, the other two rats labeled from $\mathrm{L} 1$ were judged qualitatively to have intermediate counts, and the remaining two rats labeled from $\mathrm{C} 7$ and the one labeled from $\mathrm{C} 1$ had high counts.

Summarizing the actual and qualitatively estimated counts, four of six rats labeled from $\mathrm{L} 1$ had intermediate counts, two had low counts (one was just below intermediate); four rats labeled from C7 had high counts although one was just above intermediate; and three rats labeled from $\mathrm{Cl}$ had high counts.

The total numbers of CS neurons whose axons reached various levels of the spinal cord were estimated from cross sections of the cord. Assigning the value of 1 to the area of the CS tract at the L1 level, the areas at T4, $\mathrm{C} 7$, and $\mathrm{C} 1$ were $3,3.5$, and 5 , respectively. (For comparison the cross section of the pyramid in midmedulla was 17.) From these figures and Bernstein's (4) count of about 6000 axons in each CS tract in the thoracic cord (T8), there would be about 2000 axons at $\mathrm{L} 1$ and 10,000 at $\mathrm{C} 1$. There would therefore be about $10,000 \mathrm{CS}$ neurons in each cortex whose axons reached at least the $\mathrm{C} 1$ level. Thus the method as presently used labeled a large proportion of the expected numbers of CS neurons, and despite the large variance, the numbers of neurons labeled paralleled, in rough proportion, the number of $\mathrm{CS}$ axons at each cord level.

Distribution within the Caudal Band of Neurons Labeled from Lumbar or Cervical Levels. Besides the differences in total numbers of CS neurons labeled from lumbar and cervical levels, there were also minor deviations from a generally even distribution in the caudal band of these two classes of neurons, those that sent their axons as far as the lumbar cord and those that sent them only to thoracic and cervical levels. As the lateral border of the band was approached in brains labeled from $\mathrm{Ll}$, there was a gradual decrease of labeled neurons to zero for approximately the last one-half millimeter, and sometimes the neurons disappeared short of the borderline, slightly narrowing the band. A similar decrease, but of lesser 
degree, occurred at the posterior and anterior borders. In contrast, high and intermediate counts of neurons labeled from $\mathrm{C} 1$ and $\mathrm{C} 7$ persisted as far as the lateral borderline, the labeled neuron population ending rather abruptly; the posterior and anterior borders were less abruptly defined, however. These findings indicated that there was a narrow marginal zone along the lateral border, and to a lesser extent the posterior and anterior borders, of the caudal band in which the labeled neurons were predominantly of the class whose axons reached only as far as the thoracic and cervical levels. A large proportion of these would be presumed to be concerned with forelimb movements.

Another unevenness in distribution of neurons labeled from cervical and lumbar levels was suggested by an apparent concentration of neurons labeled from L1 in a longitudinal zone of the caudal band, its middle third, extending from the posterior to anterior borders. Although the variance in our results between animals did not permit precise quantitative comparisons, counts of neurons labeled from L1 in the brain with the highest total number of neurons showed more than 100 neurons in some sagittal sections from this midzone. Sections from the same zone in brains with the highest total counts labeled from cervical levels, which included axons extending to lumbar levels, showed counts only moderately higher.

Other Consequences of IIorseradish Peroxidase Labeling. Variable labeling occurred among neurons in the red nucleus, interstitial nucleus of Cajal, vestibular nuclei, reticular formation of the medulla, and the dorsal gray matter of the cord (Rexed's laminas III to VII, approximately). As in CS neurons, the cell bodies contained relatively large discrete granules of reaction products of $\mathrm{HRP}$ with $\mathrm{DAB}$ and benzidine. At the site of cord transection and introduction of HRP, reaction products appeared in local phagocytes. Although there was a faint background coloring with DAB (but not benzidine) in the thalamus and striatum in unmagnified sections, no neurons rostral to the midbrain other than the cortical neurons were labeled. The overall pattern did not suggest labeling by blood-borne HRP or other adventitious factors. Close to the wound, axons, which were often swollen, contained the reaction products, but this was not seen at distances from the labeling site. Necrosis of neurons immediately adjacent to the wound was observed.

\section{DISCUSSION}

The principal finding was that CS neurons whose axons reached the lumbar spinal cord and those whose axons extended only to thoracic and cervical levels were generally intermixed throughout the caudal band domain. Although there were minor deviations from this configuration (such as concentration of neurons whose axons extended only to cervical and 
thoracic levels along the lateral, posterior, and anterior borders of the band), the distribution did not conform to forelimb and hindlimb motor areas determined electrophysiologically (7). The anatomic and electrophysiologic data need not seem to be in conflict, but rather should suggest experiments to find how they interrelate.

The HRP experiments did not provide information about branching axons except to indicate that the axons of about one-fifth of the CS neurons reaching the rostral cervical spinal cord continued on to lumbar levels. It is quite possible that most, even all, of these latter CS neurons gave off branches at cervical and thoracic levels $(3,15)$.

The findings of CS neurons in the rostral frontal cortex (rostral band) whose axons projected as far as $\mathrm{C} 1$ and especially $\mathrm{C} 7$ was unexpected. Previous extensive fiber degeneration studies with the Nauta method (8) showed that a very few axons from the frontal half of the motor-sensory cortex reached the cervical-medullary junction, but none was obscrved caudal to this level. In the circumstances, the HRP method seemed to be more sensitive for detecting those neurons. Preliminary studies $(9,10)$ showed that the separate rostral and caudal bands were the result of a process in which more CS neurons developed in infancy than were finally incorporated into the system. In the infant rat, the gap between the bands was well populated with neurons that could be labeled from their cut axons in the cervical cord. These axonal projections disappeared in the third week; there was no evidence, however, that this was caused by death of the cells.

The rostral band corresponds to areas from which Hall and Lindholm (7) elicited movements of jaws, lips, and tongue. In view of the mounting evidence that CS neurons, through their axonal branchings, may influence muscles of widely separated parts of the body $(3,15)$, these neurons might be concerned with coordination of mouth with forelimb movements.

\section{REFERENCES}

1. Abercrombie, M. 1946. Estimation of nuclear populations from microtome sections. Anat. Rec. 94 : 239-247.

2. Asanuma, H. 1975. Recent developments in the study of the columnar arrangement of neurons within the motor cortex. Physiol. Rev. 55 : 143-156.

3. Asanuma, H., Y. S. Shinoda, and P. Zarzecki. 1976. Branching of corticospinal fibers in the monkey. Neurosci. Abst. 2: 537.

4. Bernstein, J. J. 1966. Relationship of corticospinal tract growth to age and bsdy weight in the rat. J. Comp. Neur. $127: 207-218$.

5. Brooks, V. B., AND S. D. Stoney. 1971. Motor mechanisms: the role of the pyramidal system in motor control. Ann. Rev. Physiol. 33 : 337-392.

6. D'Amato, C. J., AND S. P. Hicks. 1975. Non-somatotopic organization of the developing motor-sensory cortex. Neurosci. Abst. $1: 784$.

7. Hall, R. D., ANd E. P. Lindirolm. 1974. Organization of motor and somatosensory neocortex in the albino rat. Brain Res. $66: 23-38$. 
8. Hicks, S. P., ANd C. J. D'Amato. 1975. Motor-sensory cortex-corticospinal system and developing locomotion and placing in rats. Am. J. Anat. 143: 1-42.

9. Hicks, S. P., And C. J. D'Amato. 1976. Labeling corticospinal and brainstem neurons in mature, infant, and radiation-malformed rats by retrograde axonal transport of horseradish peroxidase (abstract). Proc. Ann. Meeting Michigan Chapter, Society for Neuroscience, Ann Arbor, Michigan.

10. Hicks, S. P., AND C. J. D'Amato. 1976. Locating corticospinal neurons in mature, infant, and malformed (radiation) rats with retrograde axonal transport of horseradish peroxidase. Neurosci. Abst. $2: 23$.

11. König, F. R., And R. A. Kutppel. 1963. The Rat Brain. A Stereotaxic Altas. Williams and Wilkins, Baltimore.

12. KRIEG, W. J. S. 1946a. Accurate placement of minute lesions in the brain of the albino rat. Q. Bull. Northwestern Univ. Med. School. $20: 199-208$.

13. KRIEc, W. J. S. 1946b. Connections of the cerebral cortex. T. The altino rat. A. Topography of the cortical areas. B. Structure of the cortical areas. J. Comp. Neur. 84 : 221-323.

14. LAVAIL, J. H. 1975. The retrograde transport method. Fed. Proc. 34 : 1618-1624.

15. Petersen, B., Ed. 1975. Symposium on axonal branching and divergence in descending motor pathways: ABzug, C., Branching of vestibulospinal axons. Humphrey, D. H., Branching of pramidal tract axons. Petersen, B., Branching of reticulospinal axons. Shinod, Y., ANd H. Asanuma, Branching of corticospinal axons. 5th Annual Mecting Socicty for Neurosicnce, New York.

16. Porter, R. 1973. Functions of the mammalian cerebral cortex in movement. Prog. Neurobiol. 1 (part 1) : 3-51.

17. Welker, C. 1976. Receptive fields of barrels in somatosensory neocortex of the rat. J. Comp. Neur. 166: 173-190.

18. Woolsey, C. N. 1952. Patterns of localization in sensory and motor areas of the cerebral cortex. Pages 193-206 in The biology of mental health and discase. Twenty-seventh Annual Conference of the Milbank Memorial Fund, Hoeber, New York. 